\title{
Observation of the Spatial Profile of Deuterium/Hydrogen Ratio Using Bulk Charge Exchange Emission
}

\author{
Kotaro YAMASAKI, Katsumi IDA ${ }^{1)}$, Mikirou YOSHINUMA ${ }^{1)}$ and Tatsuya KOBAYASHI ${ }^{1)}$ \\ Research Institute for Applied Mechanics, Kyushu University, Kasuga Kohen, Kasuga, Fukuoka 816-8580, Japan \\ ${ }^{1)}$ National Institute for Fusion Science, Toki 509-5292, Japan
}

(Received 3 July 2018 / Accepted 22 July 2018)

\begin{abstract}
Spatial profile of deuterium/hydrogen $(\mathrm{D} / \mathrm{H})$ ratio is observed using the charge exchange emission of bulk deuterium/hydrogen ion and neutral beam perpendicularly injected into the torus plasma. Neutral beam injection (NBI) is turned on and off in order to subtract the deuterium/hydrogen emission from the plasma edge. The number of fitting parameters for the subtracted spectral signal is reduced from 12 to 3 by assuming that the bulk ion temperature and velocity are the same as those of the carbon ions $\left(C^{6+}\right)$. The initial result observed in Large Helical Device (LHD) plasma indicates that $\mathrm{D} / \mathrm{H}$ ratio is constant in radial direction and is the same as that observed from passive spectroscopic measurement when the tangential NBI or the pellet injection are absent.
\end{abstract}

(C) 2018 The Japan Society of Plasma Science and Nuclear Fusion Research

Keywords: hydrogen isotope ratio, Large Helical Device

DOI: $10.1585 /$ pfr.13.1202103

One of the current interests regarding magnetically confined fusion plasma is how the thermal/particle confinement is affected by the ratio of hydrogen isotopes [1,2]. So far, the isotope ratio is estimated from the line intensity of $\mathrm{H}_{\alpha}$ and $\mathrm{D}_{\alpha}$ [2]. In high temperature plasma, however, these line emissions mainly come from the edge of the plasma, thus the $\mathrm{D} / \mathrm{H}$ ratio observed with these signals may not represent the $\mathrm{D} / \mathrm{H}$ ratio at the core region. Recently, charge exchange emission between bulk deuterium ions and the neutral beam is used to measure the bulk ion temperature or flow [3]. In this study, charge exchange emission between bulk deuterium/hydrogen ions and the neutral beam is used to observe the $\mathrm{D} / \mathrm{H}$ ratio in the core region of the high temperature plasma. Hardware setting, the principle of the observation, and the initial result are introduced below.

Measurement is performed in Large Helical Device (LHD) [4] using perpendicular neutral beam injector (NBI\#4) [5]. Charge exchange emission is observed using tangential lines-of-sight. Schematic view of the NBI and lines-of-sight are shown in Fig. 1 (a). Observed emission is fed to optical fibers which are led to spectrometer equipped with CCD camera to observe the spectral signal. The focal length, the number of grating and the dispersion of the spectrometer are $300 \mathrm{~mm}, 2160$ grooves $/ \mathrm{mm}$ and $0.72 \mathrm{~nm} / \mathrm{mm}$, respectively. The CCD used as a detector is ANDOR IXon Ultra 897 CCD (pixel size is $16 \mu \mathrm{m} \times$ $16 \mu \mathrm{m}$ and the number of active pixels are $512 \times 512$ ). The exposure time is typically set to $5 \mathrm{msec}$. The instrumental width (FWHM) is $0.056 \mathrm{~nm}$ for the slit width of $50 \mu \mathrm{m}$ in this measurement. NBI\#4 is capable of turning on and off

author'se-mail: yamasaki@riam.kyushu-u.ac.jp

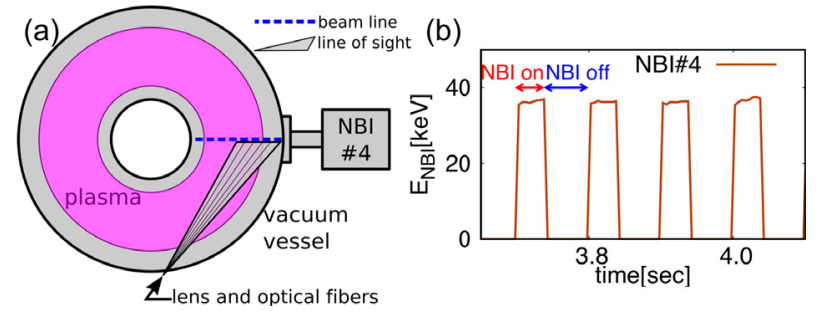

Fig. 1 (a) Position relation of perpendicular NBI and linesof-sight used for bulk charge exchange emission spectroscopy. (b) Example of the time evolution of NBI injection power. The perpendicular NBI can be turned on and off during the discharge.

the beam during the operation. An example of the waveform of NBI injection energy is shown in Fig. 1 (b).

When the neutral beam injection (NBI) is applied to the deuterium/hydrogen plasma, observed emission $I(\lambda)$ consists of the following components, excepting the emission from fast neutral beams,

$I(\lambda)$

$=I_{\mathrm{H}, \text { cold }} \exp \left[-\left(\frac{\lambda-\lambda_{\mathrm{H}, \text { cold }}}{\sigma_{\mathrm{H}, \text { cold }}}\right)^{2}\right]+I_{\mathrm{D}, \text { cold }} \exp \left[-\left(\frac{\lambda-\lambda_{\mathrm{D}, \text { cold }}}{\sigma_{\mathrm{D}, \text { cold }}}\right)^{2}\right]$
$+I_{\mathrm{H}, \text { hot }} \exp \left[-\left(\frac{\lambda-\lambda_{\mathrm{H}, \text { hot }}}{\sigma_{\mathrm{H}, \text { hot }}}\right)^{2}\right]+I_{\mathrm{D}, \text { hot }} \exp \left[-\left(\frac{\lambda-\lambda_{\mathrm{D}, \text { hot }}}{\sigma_{\mathrm{D}, \text { hot }}}\right)^{2}\right]$,

where $I_{\mathrm{H} / \mathrm{D} \text {,cold/hot }}, \lambda_{\mathrm{H} / \mathrm{D} \text {, cold/hot }}$ and $\sigma_{\mathrm{H} / \mathrm{D} \text {,cold/hot }}$ represent the intensity, the central wavelength, and $1 / e$ half width of the spectrum, respectively. The subscript $\mathrm{H}$ and $\mathrm{D}$ indicate that the value is for hydrogen or deuterium. The parameters with the subscript cold and hot indicate that the component comes from the emission from the edge 
plasma or the core region, respectively. In this study, the deuterium ratio $r_{\mathrm{D}}$ is determined from the intensity ratio of bulk charge exchange emission intensity, namely, $r_{\mathrm{D}}=I_{\mathrm{D} \text {,hot }} /\left(I_{\mathrm{H} \text {,hot }}+I_{\mathrm{D} \text {,hot }}\right)$. Since the cold component comes from the edge of the plasma, the intensity of the cold components is much stronger than that of the hot component, typically 30 times stronger. In order to subtract the huge cold component, the spectral signal obtained when NBI is turned off is adopted as the cold component and is subtracted from the spectral signal when NBI is turned on. Comparison of the spectral signal when the NBI is on and off is shown in Fig. 2 (a), and the subtracted signal is shown in Fig. 2(b). The peaks at $656.1 \mathrm{~nm}$ and $656.3 \mathrm{~nm}$ represent the residual cold component, and the broad spectrum around them represent the bulk charge exchange emission from the core region. The twelve fitting parameters have to be determined in order to fit Eq. (1) to the subtracted spectral signal. In this study, the following assumptions are applied to reduce the fitting parameters:

1. central wavelength, $1 / e$ half width and intensity ratio of the cold components are the same as those obtained from the line-of-sight which does not intersect with the core plasma region. Since the magnetic field strength is $B=2.75 \mathrm{~T}$ in this experiment, typical wavelength shift of $\mathrm{H}_{\alpha}$ due to Zeeman effect is $\Delta \lambda_{\mathrm{H} \alpha}=0.055 \mathrm{~nm}$, which corresponds to the FWHM of $4.8 \mathrm{eV}$ hydrogen spectrum, the Zeeman effect on the central wavelength can be neglected.

2. temperature and velocity of bulk deuterium/hydrogen are the same as those of carbons, namely, $\sigma_{\mathrm{H} \text {,hot }}=$ $\left(\lambda_{\mathrm{H} \alpha} / m_{\mathrm{H}}\right)\left(m_{\mathrm{C}^{6+}} / \lambda_{\mathrm{C}^{6+}}\right)\left(\sigma_{\mathrm{C}^{6+}}^{2}-\sigma_{\text {inst }}^{2}\right)+\sigma_{\text {inst }}^{2}, \sigma_{\mathrm{D}, \text { hot }}=$ $\left(\lambda_{\mathrm{D} \alpha} / m_{\mathrm{D}}\right)\left(m_{\mathrm{C}^{6+}} / \lambda_{\mathrm{C}^{6+}}\right)\left(\sigma_{\mathrm{C}^{6+}}^{2}-\sigma_{\text {inst }}^{2}\right)+\sigma_{\text {inst }}^{2}, \lambda_{\mathrm{H}, \mathrm{hot}}-$ $\lambda_{\mathrm{D}, \text { hot }}=\lambda_{\mathrm{H}_{\alpha}}-\lambda_{\mathrm{D}_{\alpha}}, \lambda_{\mathrm{H}, \text { hot }}-\lambda_{\mathrm{H}_{\alpha}}=\left(\lambda_{\mathrm{H} \alpha} / \lambda_{\mathrm{C}^{6+}}\right)\left(\lambda_{\mathrm{C}^{6+}}^{\star}-\right.$ $\lambda_{\mathrm{C}^{6+}}$ ), where $\lambda_{\mathrm{H}_{\alpha}}, \lambda_{\mathrm{D}_{\alpha}}, \lambda_{\mathrm{C}^{6+}}$, and $\lambda_{\mathrm{C}^{6+}}^{\star}$ indicate the wavelength of $\mathrm{H}_{\alpha}, \mathrm{D}_{\alpha}, \mathrm{C}^{6+}$, and observed central wavelength of $\mathrm{C}^{6+}$, respectively. Also, $\mathrm{m}_{\mathrm{H}}, \mathrm{m}_{\mathrm{D}}, \mathrm{m}_{\mathrm{C}^{6+}}$,

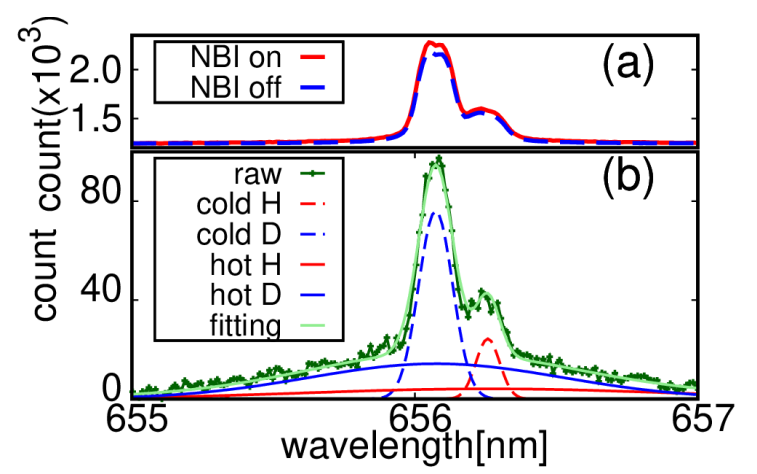

Fig. 2 Bulk charge exchange emission spectrum observed in LHD discharge 133368. (a) Spectral signals during NBI are turned on and off. (b) Example of the spectral signal obtained by subtracting the NBI off phase spectral signal. Red and blue lines indicate the hydrogen and deuterium components, and dashed line and solid line indicate the cold component and the hot component, respectively.
$\sigma_{\mathrm{C}^{6+}}$ and $\sigma_{\text {inst }}$ indicate the mass of hydrogen, deuterium, carbon, $1 / e$ width of $\mathrm{C}^{6+}$ spectrum, and that of instrumental width, respectively.

These assumptions reduce the fitting parameters to 3 , namely, $I_{\mathrm{H} \text {,cold }}, I_{\mathrm{H} \text {,hot }}$, and $I_{\mathrm{D} \text {,hot }}$. Example of fitting calculation is shown in Fig. 2(b). Fitting result is shown as the light green line and each component of Eq. (1) is plotted with red/blue solid/dashed lines. In this case, $T_{\mathrm{i}}=1.21 \mathrm{keV}, V_{\phi}=3.67 \mathrm{~km} / \mathrm{s}$, and $I_{\mathrm{D} \text {,cold }} / I_{\mathrm{H} \text {,cold }}=3.12$ are used for the fitting calculation and the obtained parameters are $I_{\mathrm{H} \text {,cold }}=24.4, I_{\mathrm{H} \text {,hot }}=3.90$ and $I_{\mathrm{D} \text {,hot }}=14.4$, resulting in $r_{\mathrm{D}}=0.78$. This calculation is adapted to all of the tangential line of sight signals to obtain $r_{\mathrm{D}}$ profile as shown in Fig. 3. The measurement result indicates that the deuterium ratio is almost constant and the same as that obtained from the passive spectroscopic measurement when tangential NBI injection or pellet injection are absent.

To summarize, bulk charge exchange emission spectroscopy was applied to observe the radial profile of $\mathrm{D} / \mathrm{H}$ ratio in LHD. $\mathrm{H}_{\alpha}$ and $\mathrm{D}_{\alpha}$ emission from the edge of the plasma are subtracted by turning on and off the perpendicular NBI during discharge. Using the information of edge plasma emission and assuming that the bulk ion temperature and flow are equal to those of $\mathrm{C}^{6+}, 4$ Gaussian fitting is performed with 3 parameters. Using these assumptions, the radial profile of $\mathrm{D} / \mathrm{H}$ ratio is obtained and is found to be almost constant when the tangential NBI or pellet injection is not applied.

We would like to thank Dr. M. Yoshida and the JT$60 \mathrm{U}$ group for providing us two spectrometers for the bulk charge exchange spectroscopy. This work is supported by the Tokamak fusion plasma joint research of National Institute for Quantum and Radiological Science and Technology (QST). This work is partly supported by JSPS KAKENHI Grant Numbers JP15H02336, JP16H02442 and JP17H01368, by the National Institute for Fusion Science grant administrative budget NIFS10ULHH021, and by the Collaborative Research Program of Research Institute for Applied Mechanics, Kyushu University.

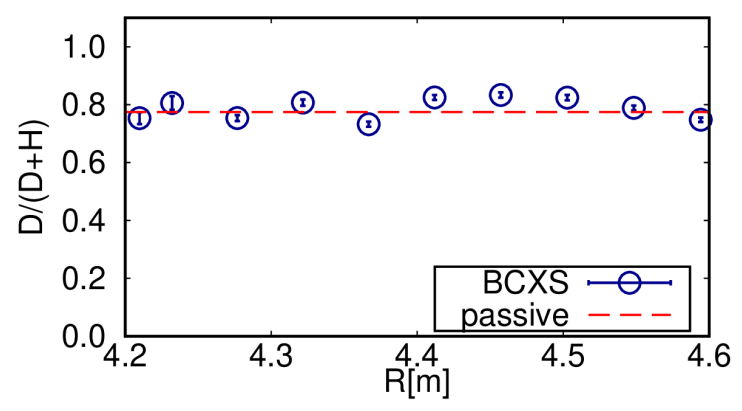

Fig. 3 Radial profile of deuterium ratio obtained from the bulk charge exchange measurement together with the calculated deuterium ratio obtained from the passive spectroscopic measurement. 
[1] G. Saibene, L.D. Horton et al., Nucl. Fusion 39, 9 (1999).

[2] Y. Xu, C. Hidalgo, I. Shesterikov et al., Phys. Rev. Lett. 110, 265005 (2013).

[3] S.R. Haskey, B.A. Grierson et al., to be published in Rev.
Sci. Instrum.

[4] Y. Takeiri et al., Nucl. Fusion 57, 102023 (2017).

[5] Y. Takeiri et al., Fusion Sci. Technol. 58, 482 (2010). 